\title{
Towards reactive augmented reality exposure treatment
}

\author{
Andreas Duenser \\ CSIRO CCl \\ Hobart, Australia \\ andreas.duenser \\ @csiro.au
}

\author{
Daniel Abramovici \\ Escola Politécnica \\ da USP \\ São Paulo, Brazil \\ abramovici@usp.br
}

\author{
Mohammad Obaid \\ t2i Lab, Chalmers Univ. of \\ Technology \\ Gothenburg, Sweden \\ mobaid@chalmers.se
}

\author{
Martin Lochner \\ CSIRO CCl \\ Hobart, Australia \\ martin.lochner \\ @csiro.au
}

\begin{abstract}
Various ways for delivering exposure treatment to phobic people have been developed and investigated. These range from traditional live exposure to mixed reality exposure to fully virtual exposure approaches, each with specific advantages and disadvantages in delivering exposure of phobia inducing stimuli. In previous research we have argued for specific advantages in creating interactive augmented reality based exposure treatment. We have extended this approach by integrating psychophysiology-based sensor data to add more interactivity, or reactivity in the system. By integrating galvanic skin response data as a real-time measurement of the user's arousal, the stimulus (a virtual spider) can automatically 'react' to the user's state. Such a system offers interesting opportunities for creating new kinds of interactive and ecologically valid exposure treatment systems that can adapt to the client's treatment needs without the need for external intervention. This paper describes our proposed approach, an initial system that we have developed and a pilot study to test the feasibility of this technology.
\end{abstract}

\section{Categories and Subject Descriptors}

H.5.1 Multimedia Information Systems: Artificial, augmented, and virtual realities

\section{General Terms}

Measurement, Design, Experimentation, Human Factors.

\section{Keywords}

Augmented reality, exposure treatment, spider phobia, psychophysiology, GSR, biofeedback, affective computing.

\section{INTRODUCTION}

Computer-based systems offer novel opportunities for creating effective exposure treatment scenarios and protocols. The spectrum of exposure treatment applications reaches from online systems designed for self-help purposes [13], to mobile apps, to fully immersive virtual environments (VE).

Creating computerized fear stimuli and treatment environments offers several opportunities for extending more traditional treatment approaches. Evidence suggests that Virtual Reality Exposure Therapy VRET can be an effective alternative to traditional in vivo exposure for specific phobias [5]. However, VRET systems remove all direct real world information from the

Permission to make digital or hard copies of all or part of this work for personal or classroom use is granted without fee provided that copies are not made or distributed for profit or commercial advantage and that copies bear this notice and the full citation on the first page. To copy otherwise, to republish, to post on servers or to redistribute to lists, requires prior specific permission and/or a fee. REHAB 2014, May 20-23, Oldenburg, Germany Copyright @ 2014 ICST 978-1-63190-011-2 DOI 10.4108/icst.pervasivehealth.2014.255341 treatment scenario, in favour of a fully simulated environment.

Augmented Reality Exposure Therapy (ARET) can increase ecological validity in such scenarios by embedding virtual objects stimuli directly into the real environment [6]. This allows users to see and interact with (virtual) fear stimuli in the real environment. Thus ARET may lead to a very realistic experience that still allows full control of the virtual fear-inducing objects. Such systems also afford direct interaction with virtual stimuli that 'react' to the client's actions and behaviour. However, the latter only has partially been explored so far.

While some degree of innovative interactivity such as integrating 3D environment tracking information has been added to ARET systems [4], we argue that real-time integration of physiological feedback can add an additional interaction layer, enhancing the systems' ecological validity even further. Such a system could be a valuable tool for clinicians, and assist in delivering more effective treatment through a more adaptive and (semi-) autonomous stimulus presentation. The real-time feedback and integration of physiology signals offers new possibilities for creating treatment scenarios, including designing real-time graded exposure by automatically adapting the system to the client's current state. In this paper we present our proposed system and an initial pilot study to investigate its feasibility.

\section{Related work}

Early ARET systems for the treatment for the fear of spiders of spiders [9] or cockroaches [2] offered limited interactivity. Using printed markers virtual spiders or cockroaches were displayed with predefined basic motion, but these implementations did not offer any interactivity. Still, these systems have been found to be effective in reducing fear [2]. The PhobiAR [4] system has taken the concept of the initial systems further by creating a more naturalistic and interactive treatment environment. This was achieved by integrating a Kinect device for tracking the 3D space in front of the user, allowing the virtual spider to interact with the environment (e.g. crawl over objects) as well as allowing the user to interact with the spider (e.g. pick up the spider).

Several studies have investigated physiological response to phobic stimulation. Lueken et al. [12] found that different regions in the brain were activated in snake phobia and dental phobia and hence that the phobia types were characterised by different neural systems. Compared to dental phobia, animal phobia was associated with autonomic arousal. Kolassa et al. [11] measured electroencephalography (EEG) signals as a response to fear or neutral stimuli using a Stroop test. No emotional Stroop interference was found for phobics when identifying the colour of the fear stimulus compared to neutral stimuli. Event-related potentials (ERPs) such as P300 and P400 were enhanced (amplitude) in spider phobics, but not in non-phobics, when viewing spider pictures.

Physiological response to exposure in a VE has been studied by Mühlberger and Wiedemann [15] comparing motion simulation to 
no-motion simulation in a flight simulator. They found that subjective fear ratings, galvanic skin response (GSR) and heart rate (HR) activations were stronger in the motion simulation condition. GSR habituated with consecutive flights. Wilhelm et al. [23] used a virtual elevator simulation and showed that height phobics showed strong GSR response but only minimal HR changes. They conclude that the behavioural inhibition system (reflected in GSR changes) is activated during VR exposure, but the behavioural activation system (HR response) is not.

Understanding the users' emotional state can be a powerful tool when coupled with interactive technologies, where the computer system adapts its functionalities based on the affective state of the user [17]. Recently, research in the field of affective computing has advanced to give human-computer interfaces the ability to interpret the user's emotional state in real-time, based on extracted human behaviour patterns such as speech, gaze, gesture, facial expressions and language [22] or HR, GSR and respiration [7]. Several systems have been developed that demonstrate the benefits of interpreting physiological signals. For example, Scheirer et al. [20] presented recommendations and guidelines for using physiological measurements in the human-computer interaction field. Their work was based on an experiment that deliberately frustrated the user, in order to provoke the user's emotional state of frustration. Nacke et al. [16] present design implications to enhance game interaction by comparing a biofeedback game design of direct versus indirect physiological control. Furthermore, a review of physiological methods used in games was presented by Kivikangas [10].

Biofeedback systems have also been developed in the health domain. Repetto et al. [19] have used a combination of GSR, HR, and respiration sensors to treat generalized anxiety disorder through progressive muscle relaxation training and guided imagery. Also Chittaro and Sioni [3] proposed a biofeedback game that helps individuals with their relaxation training. This work highlights the implications and importance for the development of future affective systems. Mingyu et al. [14] developed a brain-computer interface (BCI) system to aid in the treatment of ADHD using different wavebands to steer a spaceship in a game, and Ang et al. [1] report on a BCI based on motor imagery for motor rehabilitation in stroke patients.

In summary, evidence suggests that psycho-physiological signals can be used as a means of measuring people's response to real as well as virtual fear stimuli. Furthermore, there is great potential in using these measures to create a direct, real-time feedback mechanism to users, and to create a more interactive or re-active system. Taking these considerations into account we have developed an initial system, which is described in the next section.

\section{THE REACTIVE ARET SYSTEM}

The system is based on an adapted version of the PhobiAR application which was developed at the HIT Lab NZ [4]. We have adapted the application so that psycho-physiological sensors can be used to obtain real-time system input. This allows us to change and define the displayed behaviour of the fear stimulus (in this case a spider) according to the users physiological response.

GSR and HR were measured with the Biosemi ActiveTwo amplifier. For GSR data readings, the active electrodes were attached to the fingertips of the left hand, and for HR to the front and side of the chest (please note that analysis of the HR data is out of scope of this paper). Actiview was used to check in real time the status of all of the signals that are being read by the amplifier, to set and check configuration parameters, and to record data. It also contains TCP/IP functionality for communication between applications on the same computer, as well as applications running on different computers connected through a network. Our setup used two separate computers: one for the stimulus presentation, running the PhobiAR application, and another for data acquisition and storage from the Biosemi system.

A separate module for the TCP/IP connection was added to the PhobiAR application. This module accesses the port to which Actiview is transmitting the data in real time, having access to all the measured signals (in our case, HR, GSR and Trigger messages) with the same frequency that the Biosemi amplifier is acquiring the data $(2 \mathrm{kHz})$. Upon acquiring the data, the GSR interface module manipulates the data by aggregating the 3 bytes of data acquired from the electrodes to a single variable. The values obtained from the Biosemi are in Little Endian configuration, with a resolution of $0.03125 \mathrm{nS}$, obtained trough the Analog-Digital converter. This is the GSR reading used by the remainder of the application. Depending on the mode the application is running, this GSR reading is fed into the controller of the virtual spider in the PhobiAR application.

\section{PILOT STUDY}

The aim of this first study was to test the feasibility of the system rather than it's clinical effectiveness in treating phobia. Before planning the next steps and designing a clinical system and protocol, we wanted to get a better idea if and how the system works with a low-risk population.

As this was not designed as a clinical study we only recruited participants with low to moderate fear of spiders. Participants were screened using the Fear of Spiders questionnaire (FSQ [21]; see below for more details). We based our recruitment threshold on data from Matthews et al. [13] who had found a mean score of $93.2(\mathrm{SD}=19.4$, range 55-126) in a local sample. Therefore, we only considered participants with scores lower than 93 points on the FSQ scale for our study. After screening, 19 people from our research group (researchers from different fields, as well as administrative staff) participated in the study. Participant's age ranged from 20 to $60(\mathrm{M}=37, \mathrm{SD}=16), 12$ male and 7 female.

\subsection{Design}

We compared two conditions in a simple experiment. The two conditions were the same with the exception that one of the conditions included the use of GSR sensor data to change the spider behaviour in real time. Each participant used both conditions and we counterbalanced the presentation order.

Participants placed their right hand inside a partitioned compartment, on which the Kinect camera was focused, and viewed the resulting scene on a computer monitor in front of them (Figure 1). In the NoGSR condition the GSR signal was ignored and the spider followed the set path, interacting with the real objects on the table and following the user's hand position.

The virtual spider started in the upper left corner of the workspace and followed a triangular path, stopping at certain spots, and crossing a book, a cookie plate, and the user's hand (all these objects were real, only the spider was virtual). In the GSR condition, after a waiting period of 3 minutes to stabilize the signal, a sensor reading at the start of the trial was taken as a baseline value, and the program was given a threshold value. The controller started by setting the same path as in the non-interactive condition, but whenever the GSR reading reached values higher than the defined threshold, the spider was set to run away to the 
upper left corner of the table and to wait until the GSR readings dropped again before resuming the path.
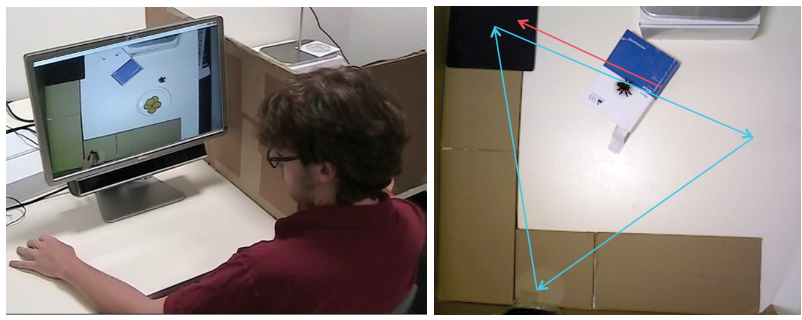

Figure 1 left: Study setup; right: spider path

\subsection{Materials}

A combination of self-reports and physiological responses (see above) was measured in order to investigate how the addition of reactivity impacts on the user. The FSQ is an 18-item scale with each item (e.g., 'If I saw a spider now, I would think it will harm me') rated on a 7-point scale ranging from 1 (definitely not) to 7 (absolutely). It was administered for screening and after each experimental session.

We also used the Subjective Units of Distress Scale (SUD), which is a scale from 0 to 10 for measuring the subjective distress currently experienced by an individual. Because there is no existing questionnaire to measure presence for these kinds of AR experiences satisfactorily, we decided to use that the Mixed Reality Experience Questionnaire (MREQ) [18], a questionnaire in development measuring various aspects of presence in mixed reality environments. Similar to de Jong et al. [8] we employed an additional question to test the perceived disgusting properties and believability of the spider. For this we put a cookie in the scene and asked the participants if they wanted to eat it, after they had seen the virtual spider crawl over it. The participants completed these three questionnaires after each experimental session.

\subsection{Results}

For the initial trials we set the GSR threshold for the spider to react and run towards the corner at $10 \%$ above the baseline. However, after several trials we realized that this value was set too high which resulted in 12 trials not showing a difference in spider behavior between the two conditions. We then reduced the threshold value to $5 \%$, which all participants reached. We decided to discard the data form the 12 trials for which the two conditions did not differ for the present analysis.

Participants in the GSR trials reached the threshold between one and seven times per trial, with an average of 3.14 times $(S D=$ 2.04). The average maximal rise in GSR signals when the threshold was recorded was $8.39 \%$ over the baseline with a maximum of $20.54 \%$. The events that preceded a threshold event were: the spider touching the book (2 times), the cookie (1) the hand (2), and starting to move after pausing (4). However, most of the threshold events could not be linked to these events (13).

For a comparison of conditions, the GSR measures were standardised to the baseline using the formula (exposure value baseline value)/SD [from baseline value]. The comparison of mean GSR level between the two conditions showed no significant differences $(t=0.77, d f=6, p=.46)$ (GSR condition $M=0.35, S D=0.61$; NoGSR condition $M=0.12, S D=0.87)$. We also compared the first session to the second session (not taking into account the sequence or actual exposure condition of these sessions) but also found no significant difference ( $t=1.01$, $d f=6, p=.35$ ).

We could not find significant differences between the two conditions in the FSQ score $(Z=-4.12, p=.68)$, the SUDs , $(Z=-$ $1.00, p=.32)$, or the cookie question, $(Z=-5.77, p=.56)$. Apart from the sub-scale 'Envelopment' (measuring the feeling of being in the same environment as the spider and feelings of disgust) all sub-scales of the MREQ (believability, spatial presence, coherence, object presence, realism, involvement) showed slightly higher ratings for the GSR condition, but none of these differences reached significance. However, the total score (all questions pooled together) was significantly different $(Z=-2.37, p$ $=.02$ ) between the two conditions. Participants gave slightly higher ratings in the GSR condition $(M=3.00, S D=0.27)$ that the NoGSR condition $(M=2.88, S D=0.22)$.

\section{DISCUSSION AND CONCLUSION}

In this work we propose creating a more interactive augmented reality-based exposure treatment system that automatically reacts to the user's arousal level. For this we designed and implemented a system that takes GSR measurements and feeds these in realtime into an ARET system. With this the virtual fear stimulus can be programed to automatically react to the user's state. In our case we explored the option of a virtual spider running away from the user once the user's GSR signal had reached a set threshold. In a study we compared this option to a condition in which the spider just follows a path irrespective of the user's reaction.

Because there is no previous data to set a suitable threshold for such a system, we had to explore different options. In our study we found an average rise of the GSR signal of around $8 \%$, however, we recognise that such threshold values heavily depend on the individuals, their fear levels and their individual reactions. Ideally such values can be established on an individual basis and be adapted to the respective needs and treatment purposes. How often people show a reaction is also very individual. We found that the threshold was reached on average three times with a relatively broad spread. Interestingly, most of the time we could not link a specific event to these reactions. Of the recorded events, the spider starting to move after pausing was the most likely cause for a GSR peak.

While we did not find a general difference in GSR measurements between the conditions, and also no difference in judged discomfort levels or fear levels, we found that overall presence ratings for the interactive GSR were significantly (though only slightly) higher. This suggests that making the spider respond to the user's arousal might increase the overall feelings of presence, including object and spatial presence, believability. However, because the questionnaire's sub scales did not show significant differences we cannot say which specific aspects of presence are affected at this stage.

We see several potential advantages of further developing such systems and eventually using them in exposure treatment contexts. Using such interactive and adaptive systems might help in achieving faster treatment outcomes because they can be designed tailored to the individual needs of patients. Exposure hierarchies can be created interactively and in real-time. A graded exposure approach could be created on-the-fly by adaptively changing the scenario and stimulus behaviour according to the reactions of the client in real-time. This can be a valuable tool for clinicians and help in reducing their workload during exposure treatment sessions by assisting in automatically adapting the treatment to the current needs of the client. Furthermore, home- 
based treatment systems could be developed that don't need much external input to present effective treatment.

There are several limitations of our current study that will have to be addressed in future research. The study did not involve a clinical sample nor a clinical protocol so we cannot yet judge the system's suitability for clinical use. Phobic people most likely would have shown other, stronger reactions to the system and stimuli. This probably would have given different results including more pronounced differences between the conditions. The current conditions might have been too similar for the chosen sample which, together with the rather small sample size, could explain why we did not find many differences in how users perceived the experiences, and why many did not reach the set threshold with the $10 \%$ setting. While suitable thresholds could be established through continued experimentation, the question remains whether it is feasible trying to find an overall threshold or whether this should better be established on an individual level.

A better approach might be to design an overall more adaptive system and change spider behaviour more dynamically. With the next version of our system we will investigate how to best implement dynamically changing the spider's distance to user's hand depending on arousal level. This for example can be achieved by defining specific regions around the hand and the less aroused the user gets, the closer the spider could move towards the hand. We also plan to investigate using other physiological signals such as HR to be integrated in the system.

\section{ACKNOWLEDGMENTS}

We thank the HIT Lab NZ for the PhobiAR application, Dr Allison Mathews, Holger Regenbrecht for the MREQ and the Brazilian CNPQ for providing a scholarship for Daniel.

\section{REFERENCES}

[1] Ang, K.K., Guan, C., Chua, K.S.G., et al. A Large Clinical Study on the Ability of Stroke Patients to Use an EEG-Based Motor Imagery Brain-Computer Interface. Clinical EEG and Neuroscience 42, 4 (2011), 253-258.

[2] Bretón-López, J., Quero, S., Botella, C., García-Palacios, A., Baños, R.M., and Alcañiz, M. An augmented reality system validation for the treatment of cockroach phobia. Cyberpsychology, Behavior, and Social Networking 13, 6 (2010), 705-710.

[3] Chittaro, L. and Sioni, R. Affective computing vs. affective placebo: Study of a biofeedback-controlled game for relaxation training. International Journal of HumanComputer Studies, (2014).

[4] Corbett-Davies, S., Dünser, A., Green, R., and Clark, A. An advanced interaction framework for augmented reality based exposure treatment. Virtual Reality (VR), 2013 IEEE, 9-22.

[5] Côté, S. and Bouchard, S. Virtual reality exposure for phobias: A critical review. Journal of CyberTherapy \& Rehabilitation 1, 1 (2008), 75-91.

[6] Dünser, A., Grasset, R., and Farrant, H. Towards immersive and adaptive augmented reality exposure treatment. Studies in health technology and informatics 167, (2011), 37-41.

[7] Fairclough, S.H. Fundamentals of physiological computing. Interacting with Computers 21, 1-2 (2009), 133-145.

[8] De Jong, P.J. and Muris, P. Spider phobia: Interaction of disgust and perceived likelihood of involuntary physical contact. Journal of anxiety disorders 16, 1 (2002), 51-65.
[9] Juan, M.C., Alcaniz, M., Monserrat, C., Botella, C., Baños, R.M., and Guerrero, B. Using augmented reality to treat phobias. Computer Graphics and Applications, IEEE 25, 6 (2005), 31-37.

[10] Kivikangas, J.M., Ekman, I., Chanel, G., et al. Review on psychophysiological methods in game research. Proc. of 1 st Nordic DiGRA, (2010).

[11] Kolassa, I.-T., Musial, F., Mohr, A., Trippe, R.H., and Miltner, W.H.R. Electrophysiological correlates of threat processing in spider phobics. Psychophysiology 0, 0 (2005)

[12] Lueken, U., Kruschwitz, J.D., Muehlhan, M., Siegert, J., Hoyer, J., and Wittchen, H.-U. How specific is specific phobia? Different neural response patterns in two subtypes of specific phobia. NeuroImage 56, 1 (2011), 363-372.

[13] Matthews, A.J., Scanlan, J.D., and Kirkby, K.C. Online Exposure Treatment for Spider Fear: The Effects of Moving Versus Static Images on Treatment Adherence, Fear Elicitation and Habituation. Behaviour Change 29, 01 (2012), 15-24.

[14] Mingyu, L., Jue, W., Nan, Y., and Qin, Y. Development of EEG biofeedback system based on virtual reality environment. Proc IEEE-EMBS. (2006), 5362-5364.

[15] Mühlberger, A. and Wiedemann, G. Subjective and Physiologic Reactions of Flight Phobics during VR Exposure and Treatment Outcome: What Adds Motion Simulation? Annual Review of CyberTherapy and Telemedicine, (2005), 185.

[16] Nacke, L.E., Kalyn, M., Lough, C., and Mandryk, R.L. Biofeedback game design: using direct and indirect physiological control to enhance game interaction. Proc. ACM CHI. (2011), 103-112.

[17] Picard, R.W. Affective Computing for HCI. Proc. of HCI International, L. Erlbaum Associates Inc. (1999), 829-833.

[18] Regenbrecht, H., Botella, C., Banos, R., and Schubert T. Mixed Reality Experience Questionnaire v1.0. Unpublished. http://www.hci.otago.ac.nz/mreq/MixedRealityExperienceQu estionnaireWeb.htm; last accessed 24/Feb/2014.

[19] Repetto, C., Gorini, A., Vigna, C., Algeri, D., Pallavicini, F., and Riva, G. The use of Biofeedback in Clinical Virtual Reality: The INTREPID Project. Journal of Visualized Experiments, 33 (2009).

[20] Scheirer, J., Fernandez, R., Klein, J., and Picard, R.W. Frustrating the user on purpose: a step toward building an affective computer. Interacting with computers 14, 2 (2002), 93-118.

[21] Szymanski, J. and O’Donohue, W. Fear of spiders questionnaire. Journal of Behavior Therapy and Experimental Psychiatry 26, 1 (1995), 31-34.

[22] Tao, J. and Tan, T. Affective computing: A review. In Affective computing and intelligent interaction. Springer, 2005, 981-995.

[23] Wilhelm, F.H., Pfaltz, M.C., Gross, J.J., Mauss, I.B., Kim, S.I., and Wiederhold, B.K. Mechanisms of Virtual Reality Exposure Therapy: The Role of the Behavioral Activation and Behavioral Inhibition Systems. Applied Psychophysiology and Biofeedback 30, 3 (2005), 271-284. 